Research Article

\title{
Heterogeneous Network Spectrum Allocation Scheme for Network-Assisted D2D Communications
}

\author{
Sungwook Kim \\ Department of Computer Science, Sogang University, 35 Baekbeom-ro (Sinsu-dong), Mapo-gu, Seoul 04107, Republic of Korea \\ Correspondence should be addressed to Sungwook Kim; swkim01@sogang.ac.kr
}

Received 16 May 2020; Revised 1 September 2020; Accepted 11 November 2020; Published 25 November 2020

Academic Editor: Adrian Kliks

Copyright (c) 2020 Sungwook Kim. This is an open access article distributed under the Creative Commons Attribution License, which permits unrestricted use, distribution, and reproduction in any medium, provided the original work is properly cited.

To meet the drastic growth of mobile traffic, next-generation wireless networks integrate existing technologies such as dualconnectivity (DC) and network-assisted device-to-device (D2D) communications. In this paper, we present a new spectrum allocation scheme for a heterogeneous system, which incorporates both technologies. For the effective collaboration of individual network agents, we employ the ideas of cooperative games, and the spectrum allocation algorithm is implemented as a novel jointbargaining process. Based on the bargaining solutions of classical Nash, weighted Nash, and Nash bargaining with coalition structure, our three-step interactive approach can leverage the full synergy of different bargaining concepts. Under the dynamic changing HetNet environments, we can take various benefits in a rational way while handling comprehensively the DC-based $\mathrm{D} 2 \mathrm{D}$ communication issue and reach an agreement that gives mutual advantage. The main novelty of our proposed scheme is to ensure a relevant tradeoff between conflicting requirements during HetNet operations. Finally, we conduct extensive simulation study and illustrate that the proposed scheme provides a considerable performance improvement by comparison with the existing protocols.

\section{Introduction}

In the last decade, the telecommunication sector has witnessed an exponential growth in the number of connected mobile devices (MDs). Therefore, mobile data traffic is growing steadily due to the prevalence of MDs along with the rapid rise in spectrum-intensive multimedia services such as mobile video streaming, mobile gaming, and social networking. According to the Cisco and Ericsson reports, the compound annual growth rate (CAGR) of overall mobile video traffic is expected to increase by $11 \%$ per year for the period of 2016-2022, and total traffic would increase by approximately six times over the period while globally reaching 8.3 billion subscriptions by 2022 . This trend is expected to thrive even faster with the introduction of upcoming sixth-generation (6G) future networks. This huge number of MDs and spectrum usage will put a heavy strain on the traditional cellular network platform, which may not provide high data rate connectivity to new applications $[1,2]$.
Currently, as a promising idea for future wireless networks, heterogeneous network (HetNet) architecture has been proposed by industry and academic researchers. It converts the traditional single cellular networks into multitier HetNets, where a growing number of small base stations (SBSs) are deployed over the existing macro base station (MBS) area; MBS and its corresponding SBSs are connected via backhaul links and tightly integrated. The dense deployment of SBSs brings the spectrum access points closer to MDs so that the traffic offloading from the MBS to SBSs may be enhanced. Global network operators, such as AT\&T, T-Mobile, Orange, and China Mobile, have widely deployed SBSs in densely populated locations such as malls, markets, and cafés to improve local capacity in traffic hotspots and relieve the burden on the overloaded MBS $[2,3]$.

Within the HetNet infrastructure, device-to-device (D2D) communication is also one of the key enabling technologies. Originally, the concept of D2D communication has been introduced to allow local peer-to-peer 
transmissions among cellular MDs bypassing the network infrastructure, such as BSs. To cope with high data rate services, the D2D communication is a promising technology to offer the following advantages: (i) extended coverage, (ii) decrease of burden from cellular networks, (iii) increased throughput, and (iv) spectrum efficiency. In particular, in 5G networks, potential D2D use-case applications have been explosively increased, that is, peer-to-peer communication, local multicasting, multiplayer gaming, data flooding, and video dissemination. However, traditional D2D technology may limit the advantages. In practice, setting up reliable direct links between the corresponding MDs is practically difficult to satisfy the quality-of-service (QoS) requirements. Usually, the potential MDs for D2D communications may not be in near proximity [4].

When MDs are too far away from each other for direct communications, network-assisted D2D communication has recently been proposed as a means for real-world operations. In the case of example, MD pairs for $\mathrm{D} 2 \mathrm{D}$ communications can be located in the different cell areas, and BSs are working as relay nodes. The cellular spectrum resources at relay BSs are assisted by a network infrastructure and shared for the D2D communication links. To leverage network-assisted D2D communications, the network system should (i) mediate in the D2D pairing process, (ii) coordinate the time and spectrum allocations, and (iii) ensure the required QoS. It can lead to increased system throughput and higher spectrum efficiency. To obtain these potential gains, there is a need to carefully design novel spectrum allocation algorithms that effectively manage the D2D pairs in cellular areas. However, to fully exploit its potential, there are some critical control issues that should be addressed to design algorithms $[4,5]$.

Among these critical issues, MD association is one of major problems; it involves the spectrum allocation process in BSs with the goal of improving the per-MD throughput and overall system capacity. Recently, the concept of dual connectivity (DC) in HetNets has been introduced to use simultaneously the spectrum resources in MBS and SBS. The DC technique constitutes a novel feature that contributes to effectively handling the spectrum demands by enabling MDs to maintain two concurrent connections. More broadly, DCbased spectrum aggregation solution can enhance $\mathrm{MD}$ connectivity experience as well as the overall communications reliability. This flexible DC technique association, supported by the interoperability of different BSs, makes a decisive step to maximize the HetNet system efficiency. To design a new HetNet spectrum allocation algorithm by taking into account all the critical issues, we need a new intelligent control paradigm and novel solution concept [6].

1.1. Technical Concepts. In the HetNet management algorithms, autonomous, distributed, and intelligent network agents independently make rational and strategic decisions. This scenario may fall into game theory. In general, game theory is based on a particular theory of rationality and offers a comprehensive analysis of rational behavior under circumstances of strategic interdependence. Therefore, it provides a formal analytical framework with a set of mathematical tools to study the complex interactions among rational game players. Throughout the past decades, game theory has made revolutionary impact on a large number of disciplines ranging from engineering, economics, political science, philosophy, or even psychology. In recent years, there has been a significant growth in research activities that use game theory for analyzing wireless network agents under competitive or collaborative situations [7].

As a subfield of game theory, bargaining game is an effective tool to achieve a mutually desirable solution with a good balance between efficiency and fairness. It comes with no surprise that bargaining solution has gained wide popularity and it still constitutes an active research strand. In 1950, Nash originally introduced the fundamental notion of the Nash bargaining solution (NBS) to share the resource fairly and optimally $[8,9]$. Based on the idea of NBS, the weighted Nash bargaining solution (WNBS) was developed. In the WNBS, bargaining powers of heterogeneous game players are unequal; they may be driven by different characteristics [7, 10]. In 2004, Chae and Heidhues proposed a novel concept of NBS with coalition structure (NBS-CS) [9]. In the NBS-CS, bargaining process is actually taking place simultaneously with different coalitions. Each coalition consists of multiple individuals, and they act cooperatively with each other.

1.2. Main Contributions. According to the NBS, WNBS, and NBS-CS, we develop a novel spectrum allocation scheme for DC-based D2D communications. In the HetNet platform, each MBS distributes the spectrum resource to its corresponding SBSs according to the concept of NBS. Based on the assigned spectrum resource, each individual SBS allocates its spectrum resource to each individual application services based on the idea of WNBS. Then, the MBS provides additionally its own spectrum resource to supplement running applications. By considering the network-assisted D2D communications, the concept of NBS-CS is applied to implement the CD technique. In the combination of NBS, WNBS, and NBS-CS, we can leverage the full synergy of different bargaining solutions while handling comprehensively DC-based D2D communication issues. In detail, the major contributions of this study are as follows:

(i) This study considers the spectrum allocation problem in the HetNet infrastructure. During the three-step interactive bargaining process, the limited spectrum resource is hierarchically distributed to multiple MDs from their corresponding SBS and MBS.

(ii) Each MBS distributes the orthogonal bandwidth portions into its covering SBSs based on the NBS. Then, each individual SBS allocates its assigned spectrum resource by using the idea of WNBS; MDs are treated differently whether MDs are involving D2D communications or not.

(iii) With the DC technique, each MBS supplementally allocates its own the spectrum resource for MDs. By 
considering the coalition for D2D-involved MDs, the concept of NBS-CS is applied, and the repeated bargaining process is used to allocate the MBS resource.

(iv) Under hierarchical HetNet platform, we explore the interaction of different bargaining solutions while leveraging the synergistic features. The main characteristic of our joint-bargaining approach lies in its responsiveness to the reciprocal combination of NBS, WNBS, and NBS-CS.

(v) Numerical results are shown to give the comparisons and performance gains between the proposed scheme and the existing protocols. With respect to different performance criteria, we can enhance the overall system performance in the HetNet infrastructure.

1.3. Organization. The remainder of our paper is organized as follows. In Section 2, we provide a literature overview that covers the prior spectrum allocation algorithms in networkassisted D2D communications. In Section 3, we describe the DC-based HetNet system model and its underlying assumptions to formulate the spectrum allocation problem. Then, we introduce the basic ideas of NBS, WNBS, and NBSCS to design our HetNet spectrum management scheme. Based on the joint-bargaining approach, the main steps of our proposed algorithm are given to increase readability. The simulation setup and comprehensive performance comparisons with other existing protocols are provided in Section 4. For the readers' facilitation, experimental results are provided along with discussions. Finally, Section 5 summarizes the main contributions and conclusions in this study. In addition, some future research directions and issues are also outlined in this section.

\section{Related Work}

Since the initial concept of network-assisted D2D communications was introduced, state-of-the-art studies have been conducted on spectrum efficiency in the HetNet architecture. The study in [11] proposes a new cluster-based cognitive industrial Internet of Things (CIIoT) to improve spectrum utilization by accessing the idle spectrum licensed to a primary user (PU). Cooperative spectrum sensing among the cluster heads and nonorthogonal multiple-access-based transmission in each cluster are also proposed to improve both sensing probability and transmission performance of the CIIoT. To maximize the average total throughput of the CIIoT, joint resource allocation of sensing time, node powers, and the number of clusters are presented. This approach can guarantee the minimal detection probability, the maximal total power, and the minimal rate of each node [11].

In [12], a novel CIIoT control scheme is implemented to harvest the radio frequency energy of PU's signal. In addition, energy-efficient resource allocation algorithms in different spectrum access modes are also presented to maximize the average transmission rate of CIIoT while guaranteeing its energy-saving requirements. They are formulated as corresponding optimization problems that seek to maximize the average transmission rate of CIIoT under the constraint of energy-saving. The optimization problems are solved by using the alternating direction optimization and water-filling algorithm [12].

In [13], the No-Regret Bandit Learning (NRBL) scheme is proposed to address the distributed channel selection problem for network-assisted D2D communications. Usually, individual D2D users compete for access to the spectrum resources without governing by any centralized controller. This situation, as it appears in many wireless networking scenarios, can be modelled as a multiplayer multiarmed bandit game with side information, and selfish D2D users aim at optimizing their own performance by using the vacant cellular channels. The NRBL scheme provides a new distributed algorithmic solution, which is a combination of no-regret learning and calibrated forecasting. For each D2D user, the long-term average reward is equal to the average reward of the optimal selection. To get the solution, selfish D2D users learn an optimal joint action profile from successive interactions with a dynamic environment and finally settle at some equilibrium points, such as the set of correlated equilibria [13].

The Statistical Quality-guaranteed Spectrum Interoperation (SQSI) scheme is developed for network-assisted D2D communications while alleviating the spectrum scarcity issue of cellular networks [1]. For network-assisted interoperable D2D communications, the SQSI scheme brings about many benefits including the higher D2D users' throughput and better network management. In addition, the issue of ensuring QoS is investigated under dynamic network environments. In particular, the SQSI scheme analyzes the QoS issue using a well-known analytical tool, "Effective Capacity (EC)," for the network operability. According to the impact of neighboring cells' load, the ECbased statistical QoS guarantee analysis has been carried out under varying channel conditions and provides statistical QoS guarantees for the spectrum interoperable D2D communications. Under varying channel conditions along with different cell load, the SQSI scheme can calculate the maximum sustainable source rate at the transmitter's queue. The simulation results indicate that a better cell load management can lead to more appropriate system performance [1].

Liu et al. propose the Small cell based Distributed Resource Allocation (SDRA) scheme for D2D-assisted heterogeneous networks [14]. By taking the required data rate of D2D users, they formulate a channel allocation problem, which is integrating the channel selection and channel sharing processes to maximize the service satisfaction of all D2D users. In the considered system model, a heterogeneous spectrum pool consisting of different bands is provided for the access channel selection. To derive the solution, they provide two games: a potential game by using an interference graph and a coalition game with D2D user transferring. In the potential game, they get the stable matching between different users and channels in different frequency bands. In the coalition game, the final coalitions between users are 
realized through D2D user transferring. Based on these two games, a two-stage distributed channel allocation algorithm is designed with low computational complexity. Finally, simulation results have demonstrated that the SDRA scheme can achieve a higher system throughput performance and a better network utility [14].

Although a lot of researches have exploited extensively the network-assisted D2D technique in HetNets, an efficient integration of DC operations with the HetNet platform has not been fully utilized. To the best of the author's knowledge, this is the first study that provides a fair-efficient spectrum allocation solution for the DC-based interoperable D2D communication. By taking temporal HetNet traffic fluctuations into account, we make rational decisions in the threestep bargaining process and jointly allocate the limited spectrum resource to multiple MDs. Different from existing NRBL, SQSI, and SDRA protocols, our proposed scheme can reach an agreement that gives mutual advantage and has more potential benefits in terms of MD's payoff, system throughput, and fairness among BSs.

\section{The Bargaining-Based Spectrum Allocation Algorithm}

In this section, the purpose of DC-based network-assisted D2D communications and the basic concept of cooperative games have been studied. According to the three-step jointbargaining approach, we design our spectrum allocation protocol while discussing relevant control issues. Finally, the main step procedures of our proposed algorithm are delineated to help readers have better understanding.

3.1. DC-Based Heterogeneous Network System Infrastructure. In this study, we consider a two-tier heterogeneous network platform, which comprises two types of BSs with orthogonal spectrum resource pools. At the upper tier, MBSs $\mathscr{M}=\left\{\mathscr{M}_{1}, \ldots, \mathscr{M}_{2}\right\}$ exist, and they can provide cellular network coverage for a large area. Within the coverage of each $\mathscr{M}$, multiple SBSs break up a macrocell site into much smaller areas. Therefore, SBSs $\mathcal{S}=\left\{S_{1}, \ldots, S_{m}\right\}$ coexist at the lower tier, and the cross-tier DC transmission is provided. One MBS (or one SBS) has a coverage area of radius $r_{M}$ (or $r_{S}$ ) and has a static portion of spectrum resource. It is more practical and suitable for the scenario where the different serving levels are ensured over different association spectrum bands. In this paper, we assume that each MBS has its own spectrum resource $\left(\mathfrak{R}_{M}\right)$ and the spectrum resource $\left(\mathscr{M}_{M}\right)$ for SBSs; $\mathscr{M}_{M}$ is adaptively distributed into corresponding SBSs. Afterward, individual SBSs also divide orthogonally their assigned spectrum pool into a set of different channels to support diverse applications [15].

In the HetNet cellular area, there are multiple MDs $\mathscr{D}=\left\{D_{1}, \ldots, D_{k}\right\}$; they are assumed to be randomly distributed and are equipped with two different radio interfaces to communicate simultaneously with the MBS and SBS. With a dynamic monitoring for the MBS and SBS, the DCenabled traffic scheduling is expected to further enhance the data capacity and overall network efficiency. Besides this, some MDs are paired and transmit data to their corresponding MDs by using the D2D communication mode. In this mode, the paired MDs share their status information in a timed manner. In the emerging $5 \mathrm{G}$ networks, MBSs work as software-defined network (SDN) controllers for both cellular and D2D connections. Motivated by the fact that BSs generally have whole network information and high computation power, we assume that the BSs determine the task schedule for MDs at each time frame while effectively managing the spectrum resource. Therefore, during the DCbased HetNet operation, the complex interaction between the D2D pair is modelled with their corresponding BSs [16].

In this study, a modelling situation for the HetNet spectrum allocation process is formulated as a joint-bargaining game $(\mathscr{G})$ in a cooperative manner. The HetNet system operates in a slotted structure and each time frame has a time length $t$. Formally, we define game entities, that is, $\mathscr{G}=\left\{\{\mathscr{M}, \mathcal{S}, \mathscr{D}\},\left\{\mathfrak{R}_{M_{i} \in M^{\prime}} \mathscr{M}_{M \in \mathscr{M}}\right\}, \mathscr{X}_{D z D},\left\{U_{M_{i}}, U_{S_{j}}^{M_{i}}, U_{D e}^{M_{j}}\right\}\right.$, $T\}$, at each time period of gameplay.

(i) $\{\mathscr{M}, \mathcal{S}, \mathscr{D}\}$ are the finite set of MBSs, SBSs, and MDs; they are game entities for our joint-bargaining games.

(ii) $\Re_{M_{i}}$ and $\mathscr{M}_{M_{i}}$ are the spectrum amount assigned for $M_{i} \in \mathscr{M} ; \Re_{M_{i}}$ is used by $M_{i}$ for the DC services, and $\mathscr{M}_{M_{i}}$ is distributed for $S_{j} \in \mathscr{W}_{M_{i}} \subset \mathcal{S}$, where $\mathscr{W}_{M_{i}}$ is the set of SBSs within $M_{i}$ 's area.

(iii) $\mathscr{X}_{D z D}$ is the set of MDs, which are involved in the network-assisted D2D communications. Therefore, each of its elements is paired with other elements in $x_{D z D}$.

(iv) $U_{M_{i}}, U_{S_{j}}^{M_{i}}, U_{D_{e}}^{S_{j}}$ are utility functions of $M_{i}, S_{j}$, and $D_{e}$, respectively, where $S_{j} \in \mathscr{W}_{M_{i}}$ and $D_{e}$ is in $S_{j}$ 's covering area.

(v) $T=\left\{t_{1}, \ldots, t_{c}, t_{c+1}, \ldots,\right\}$, where $T$ denotes time, which is represented by a sequence of time steps.

3.2. The Basic Concepts of Cooperative Bargaining Solutions. Many HetNet situations can be modelled by a set of network agents which may benefit from cooperation. Therefore, it is not infrequent that these agents do not act individually but are partitioned into coalitions. Assuming that HetNet agents' cooperation is carried out, one may wonder how the benefit is shared between the coalitions and between the agents inside each coalition. Based on the generalization of Nash solution, different bargaining models can address this issue. In the standard cooperative game paradigm, a bargaining problem refers to sharing a surplus that game players can jointly generate. Therefore, bargaining solutions study how the surplus should be shared with appealing axioms [7, 9].

To define the basic idea of bargaining solution, we introduce the notation and preliminary definitions of bargaining solution. Let $\mathbf{N}$ be a finite set of players. We denote by $|\mathbf{N}|$ the number of elements of $\mathbf{N}$. Let us take $x, y \in \mathbb{R}^{\mathbf{N}}$. We say $y \leq x$ when $y_{i} \leq x_{i}$ for each $i \in \mathbf{N}$ and $y<x$ when $y_{i}<x_{i}$ for each $i \in \mathbf{N}$. We denote by $(x \cdot y)$ the vector $\left(x_{i} \cdot y_{i}\right)_{i \in \mathbf{N}}$ and by $(x+y)$ the vector $\left(x_{i}+y_{i}\right)_{i \in \mathbf{N}}$. Given 
$T \subseteq \mathbf{N}, x_{T}$ is the restriction of $x$ to $\mathbb{R}^{T}$. We denote by $\mathbb{R}_{+}^{\mathbf{N}}$ the set $\left\{x \in \mathbb{R}^{\mathbf{N}}: x_{i} \geq 0\right.$ for every $\left.i \in \mathbf{N}\right\}$ and by $\mathbb{R}_{++}^{\mathbf{N}}$ the set $\left\{x \in \mathbb{R}^{\mathbf{N}}: x_{i}>0\right.$ for every $\left.i \in \mathbf{N}\right\}$. Given $\gamma \in \mathbb{R}_{++}^{\mathbf{N}},(1 / \gamma)$ is the vector $\left(1 / \gamma_{i}\right)_{i \in \mathrm{N}}[8,9]$.

A feasible set $\mathbf{S}$ is a subset of the payoff space, and a disagreement point $d$ is an element of the payoff space. The points in $\mathbf{S}$ represent the feasible utility levels that the individuals can reach if they agree. Otherwise, if agreement is not reached, they obtain the utility levels given by the disagreement point. For every $\mathbf{S} \subseteq \mathbb{R}^{\mathbf{N}}$ and $\gamma, \beta \in \mathbb{R}^{\mathbf{N}}$, we define $(\gamma \cdot \boldsymbol{S})+\beta=\{(\gamma \cdot x)+\beta \mid x \in S\}$. Given $\theta \in \mathbb{R}$ and $x \in \mathbb{R}^{\mathbf{N}}$, we define $(\theta \cdot x)$ as the vector $\left(\theta \cdot x_{i}\right)_{i \in \mathbf{N}}$. A coalition structure $\mathscr{C}$ over $\mathbf{N}$ is a partition of the player set; that is, $\mathscr{C}=\left\{C_{1}, \ldots, C_{p}\right\} \subseteq 2^{\mathbf{N}}$, where $\cup_{\left(C_{q} \in \mathscr{C}\right)} C_{q}=\mathbf{N}$ and $C_{q} \cap C_{r}=$ $\varnothing$ whenever $q \neq r$. Each $C_{q} \in \mathscr{C}$ is called a coalition. We denote by $c \in \mathbb{R}^{N}$ the vector whose $i^{\text {th }}$ coordinate is given by $c_{i}=\left|C_{q}\right|$ if $i \in C_{q}$. Usually, bargaining game is a pair $(\mathbf{N}, v)$, where $v$ is a characteristic function that assigns to each subset $T \subseteq \mathbf{N}$ a number $v(T) \in \mathbb{R}$, with $v(\phi)=0 . v(T)$ represents the total utility payoff when players in $T$ can cooperate. A bargaining problem over $\mathbf{N}$ is a pair $(\mathbf{S}, d)$, where $d \in \mathbf{S} \subseteq \mathbb{R}^{\mathbf{N}}$. Let the boundary of $\boldsymbol{S}$ be $\partial S$, and there exists $x \in \mathbf{S}$ such that $x>d$. The traditional Nash bargaining solution is the unique point $\mathbf{N}(\mathbf{S}, d) \in \partial S$ satisfying the following equation $[8,9]$ :

$$
\prod_{i \in \mathbf{N}}\left(\mathbf{N}_{i}(\mathbf{S}, d)-d_{i}\right)=\max _{x \in \mathbf{S}, x \geq d} \prod_{i \in \mathbf{N}}\left(x_{i}-d_{i}\right) .
$$

In equation (1), all the players involved in the bargaining game will be assigned the same bargaining powers. It may not be reasonable when the players are significantly different. As a generalized Nash bargaining solution, WNBS is a variant of the NBS by assigning players with different bargaining powers. To get the WNBS, the objective function in (1) is modified as follows [10]:

$$
\begin{aligned}
\prod_{i \in \mathbf{N}}\left(\mathbf{N}_{i}(\mathbf{S}, d)-d_{i}\right) & =\max _{x \in \mathbf{S}, x \geq d} \prod_{i \in \mathbf{N}}\left(x_{i}-d_{i}\right)^{\omega_{i}}, \\
\text { s.t. } \sum_{i \in \mathbf{N}} \omega_{i} & =1,
\end{aligned}
$$

where $\omega_{i}$ is the corresponding bargaining power of the player $i \in \mathbf{N}$. Bargaining power is a concept related to the relative abilities of game players in a situation to exert influence over each other. To provide additional flexibility in choosing solution by taking into consideration the diversified network situation, it is necessary to admit differential bargaining powers [7].

A bargaining game with coalition structure is a triple $(\mathbf{N}, v, \mathscr{C})$, where $\mathscr{C}$ is a coalition structure over $\mathbf{N}$, and the bargaining problem of $(\mathbf{N}, v, \mathscr{C})$ is a triple $(\mathbf{S}, d, \mathscr{C})$. A solution of $(\mathbf{S}, d, \mathscr{C})$, which is characterized by the unique point $\phi(\mathbf{S}, d, \mathscr{C}) \in \partial S$, is the NBS-CS, and it is obtained as follows [9]:

$$
\begin{aligned}
& \max _{\mathbb{U} \in S, \cup \geq \mathbf{d}} \prod_{C_{j} \in \mathscr{C}}\left(\prod_{i \in \mathbf{N}}\left(x_{i}-d_{i}\right)^{f\left(C_{j}\right)}\right) \\
& \text { s.t. }\left\{\begin{array}{l}
\mathscr{C}=\left\{C_{1}, \ldots, C_{p}\right\} \\
f\left(C_{j}\right)>0
\end{array}\right.
\end{aligned}
$$

The NBS, WNBS, and NBS-CS are characterized by a collection of desirable axioms such as invariance with respect to affine transformation (IAT), independence of irrelevant alternatives (IIA), strong individual rationality (SIR), and anonymity (AN) $[8,9]$.

(i) IIA: let a bargaining solution be a function $F$ that associates with each problem $(\mathbf{S}, d)$ or $(\mathbf{S}, d, C)$ a feasible payoff vector $F(\mathbf{S}, d)$ or $F(\mathbf{S}, d, C)$. If there exists another bargaining problem $\left(S^{\prime}, d\right)$ or $(\mathbf{S} \prime, d, C)$ such that $S^{\prime} \subseteq \mathbf{S}$ and $F(\mathbf{S}, d)$ or $F(\mathbf{S}, d, C)$ belongs to $S^{\prime}$, then $F\left(S^{\prime}, d\right)=F(\mathbf{S}, d)$ and $F\left(S^{\prime}, d, C\right)=F(\mathbf{S}, d, C)$.

(ii) IAT: given $\quad \gamma=\left\{\gamma_{1}, \ldots, \gamma_{n}\right\} \in \mathbb{R}_{++}^{\mathbf{N}} \quad$ and $\beta=\left\{\beta_{1}, \ldots, \beta_{n}\right\} \in \mathbb{R}^{\mathbf{N}}$, it holds that $F(\bar{S}, t \bar{d})=(\gamma$. $F(\mathbf{S}, d))+\beta \quad$ and $\quad F(\bar{S}, t \bar{d} n, q C)=(\gamma \cdot F(\mathbf{S}$, $d, C))+\beta$, where $\bar{S}=(\gamma \cdot \mathbf{S})+\beta$ and $\bar{d}=(\gamma \cdot d)+\beta$.

(iii) SIR: for all $i \in \mathbf{N}, F_{i}(\mathbf{S}, d, C)>d_{i}$, and $F_{i}(\mathbf{S}, d)>d_{i}$. Therefore, there is no $x \in \mathbf{S} \backslash\{F(\mathbf{S}, d, C)$ or $F(\mathbf{S}, d)\}$ such that $x_{i} \geq F_{i}(\mathbf{S}, d, C)$ and $x_{i} \geq F_{i}(\mathbf{S}, d)$.

(iv) AN: for any permutation $\phi: \mathbf{N} \longrightarrow \mathbf{N}$, $F(\phi(\mathbf{S}), \phi(\mathbf{d}), \phi(C))=\phi(F(\mathbf{S}, d, C)) \quad$ and $F(\phi(\mathbf{S}), \phi(\mathbf{d}), \phi(C))=\phi(F(\mathbf{S}, d, C))$.

3.3. The Bargaining Control Scheme for DC-Based HetNet System. In this study, we design a new spectrum management scheme for the hierarchical HetNet platform. The major goal of our scheme is to adaptively allocate the limited spectrum resource to MDs. To reduce computation complexity, the spectrum allocation process is divided hierarchically, and we make control decisions in a step-by-step manner at each stage. In the first phase, each individual MBS $(M)$ has $\mathscr{M}_{M}$ and shares it with the set of SBSs $\left(\mathscr{W}_{M}\right)$ within $M$ 's area. Therefore, $\mathscr{M}_{M}$ is partitioned into disjoint portions and allocates them for corresponding SBSs. To implement this process for $M_{i}$ with $\mathscr{W}_{M}$, we focus on the traditional NBS to get a fair-efficient solution. Let $\mathscr{X}_{S_{i}}^{M_{i}}$ be the spectrum amount allocated for the $S_{i} \in \mathscr{W}_{M_{i}}$, and $M_{i}$ 's spectrum distribution solution for $\mathscr{W}_{M_{i}}$, that is, $\Theta_{\mathscr{W}_{i}}^{M_{i}}$, is a $\left|\mathscr{W}_{M_{i}}\right|$-dimensional bandwidth allocation vector, where $\Theta_{\mathscr{W}_{M_{i}}}^{M_{M_{i}}^{M_{i}}}=\left\langle\ldots X_{S_{j} \in \mathscr{W}_{M_{i}}}^{M_{i}} \ldots\right\rangle$. 


$$
\begin{aligned}
\Theta_{\mathscr{W}_{M_{i}}}^{M_{i}} & =\underset{\left(\ldots X_{S_{j} \in \mathscr{W}_{M_{i}}}^{M_{i}} \ldots\right)}{\max }\left(\prod_{S_{j} \in \mathscr{W}_{M_{i}}}\left(U_{S_{j}}^{M_{i}}\left(\mathscr{X}_{S_{j}}^{M_{i}}\right)-d_{S_{j}}\right)\right), \\
& \text { s.t. }\left\{U_{S_{j}}^{M_{i}}\left(\mathscr{X}_{S_{j}}^{M_{i}}\right)=\left(\exp \left(\frac{\min \left(\mathscr{X}_{S_{j}}^{M_{i}}, r^{t_{c}}\left(\mathbf{N}_{S_{j}}^{M_{i}}\right)\right)}{r^{t_{c}}\left(\mathbf{N}_{S_{j}}^{M_{i}}\right)} \times \psi_{S}\right)-\mu\right)\left(\sum_{S_{j} \in w_{M_{i}}} \mathscr{X}_{S_{j}}^{M_{i}}\right) \leq \mathscr{M}^{M},\right.
\end{aligned}
$$

where $\psi_{S}$ is a profit coefficient factor and $\mu$ is a control parameter for $U_{S_{j}}^{M_{i}}(\cdot)$, respectively. $\mathbf{N}_{S_{j}}^{M_{i}}$ represents the set of MDs, which exist in $S_{j}$ 's area covered by $M_{i}$. At time $t_{c}$, $r^{t_{c}}\left(\mathbf{N}_{S_{j}}^{M_{i}}\right)$ are the total requested spectrum amounts of MDs in $\mathbf{N}_{S_{j}}^{M_{i}^{j}}$.

In the second phase, each individual $S$ allocates its assigned spectrum pool $\left(\mathscr{X}_{S}^{M}\right)$ for its corresponding MDs in
$\mathbf{N}_{S}^{M}$. In this study, the major challenge is to effectively support network-assisted D2D communications. Therefore, to implement $S$ 's spectrum allocation process, we prioritize D2D services and focus on the WNBS. Let $\Theta^{S_{j}}\left[D_{e} \in \mathbf{N}_{S_{j}}^{M_{i}}\right]=$ $\left\langle\ldots, X_{D_{e}}^{S_{j}}, \ldots\right\rangle_{S_{j}}$ be a $\left|\mathbf{N}_{S_{j}}^{M_{i}}\right|$-dimensional spectrum allocation vector, and $\mathscr{X}_{D_{e}}^{S_{j}}$ is the assigned spectrum amount for $D_{e}$. Finally, $\Theta^{S_{j}}\left[D_{e} \in \mathbf{N}_{S_{j}}^{M_{i}}\right]$ is obtained as follows:

$$
\begin{aligned}
& \Theta^{S_{j}}\left[D_{e}\right]=\max _{\ldots, \mathcal{X}_{D_{e}}^{S_{j}}, \ldots}\left(\prod_{D_{e} \in N_{S_{j}}^{M_{i}}}\left(U_{D_{e}}^{S_{j}}\left(x_{D_{e}}^{S_{j}}, r_{D_{e}}^{S_{j}}\right)-d_{D_{e}}\right)^{\omega_{D_{e}}}\right)
\end{aligned}
$$

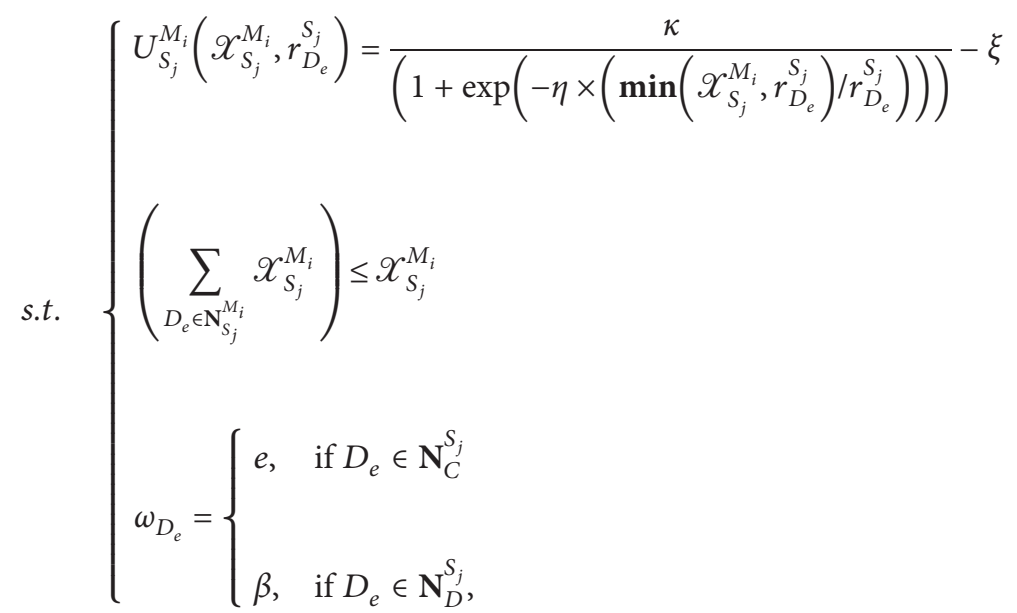

where $\kappa, \eta$, and $\xi$ are coefficient factors for $U_{D_{e}}^{S_{j}}(\cdot) . r_{D e}$ is the requested spectrum amount of $D_{e} . e$ and $\beta$ are bargaining powers for MDs. $\mathbf{N}_{C}^{S_{j}}$ is the set of simple cellular MDs and $\mathbf{N}_{D}^{S_{j}}$ is the set of network-assisted D2D MDs, where $\mathbf{N}_{S_{j}}^{M_{i}}=\mathbf{N}_{C}^{S_{j}} \cup \mathbf{N}_{D}^{S_{j}}$.

In the third phase, the MBS provides its own spectrum resource $\left(\mathfrak{R}_{M}\right)$ for DC services. Let $\mathbf{N}_{C}^{M_{i}}=\cup_{S_{j} \in \mathscr{W}_{M_{i}}} \mathbf{N}_{C}^{S_{j}}$ and $\mathbf{N}_{D}^{M_{i}}=\cup_{S_{j} \in \mathscr{W}_{M_{i}}} \mathbf{N}_{D}^{S_{j}} ; \mathbf{N}_{C}^{M_{i}} ; \mathbf{N}_{C}^{M_{i}}$ and $\mathbf{N}_{D}^{M_{i}^{C}}$ can be treated as a unit under diverse traffic environments. Therefore, the bargaining with coalition structure is applied to provide spectrum resources for DC services. Let $\varepsilon_{D_{i}}^{M_{i}}$ be an added spectrum amount supplemented by $M_{i}$ for $D_{e}$. Finally, the solution $\left(\Theta\left[D_{e} \in\left(\mathbf{N}_{C}^{M_{i}} \cup \mathbf{N}_{D}^{M_{i}}\right)\right]\right)$ is a $\left|\mathbf{N}_{C}^{M_{i}} \cup \mathbf{N}_{D}^{M_{i}}\right|$-dimensional spectrum allocation vector, where $\Theta\left[D_{e} \in\left(\mathbf{N}_{C}^{M_{i}} \cup \mathbf{N}_{D}^{M_{i}}\right)\right]=\left\langle\ldots, \varepsilon_{D_{e}}^{M_{i}}\right\rangle ; \Theta\left[D_{e} \in\left(\mathbf{N}_{C}^{M_{i}} \cup \mathbf{N}_{D}^{M_{i}}\right)\right]$ is obtained as follows: 


$$
\begin{aligned}
& \Theta\left[D_{e} \in\left(\mathbf{N}_{C}^{M_{i}} \cup \mathbf{N}_{D}^{M_{i}}\right)\right]=\max _{\substack{M_{i} \\
\left\langle\ldots D_{D_{e}} \ldots\right\rangle}} \prod_{X \in\left\{\mathbf{N}_{C}^{M_{i}}, \mathbf{N}_{D}^{M_{i}}\right\}}\left(\prod_{D_{e} \in X}\left(U_{D_{e}}^{M_{i}}\left(\varepsilon_{D_{e}}^{M_{i}}, \mathscr{X}_{D_{e}}^{S_{j}}, r_{D_{e}}^{S_{j}}\right)-d_{D_{e}}\right)^{F\left(D_{e}, D_{k}\right)}\right),
\end{aligned}
$$

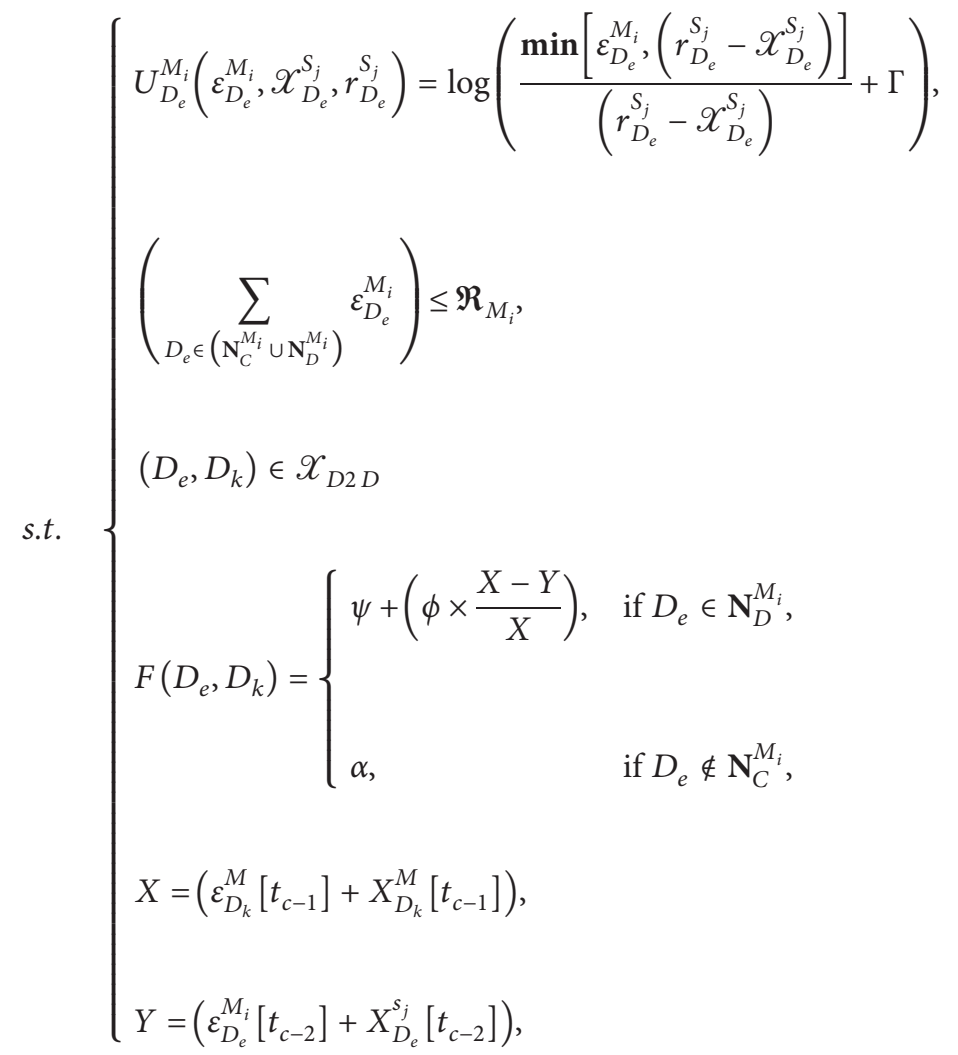

where $\Gamma$ is a control parameter for $U_{\mathscr{D}_{e}}^{\mathscr{M}_{i}}(\cdot) . \psi, \alpha$, and $\phi$ are profit coefficient factors for ${ }^{e} F\left(\mathscr{D}_{e}, \mathscr{D}_{k}\right)$ when $\mathscr{D}_{e}, \mathscr{D}_{k} \in \mathscr{X}_{D 2 D}$ and $\mathscr{D}_{k}$ is paired with $\mathscr{D}_{e}$ for D2D communications. $\varepsilon_{D_{k}}^{M}\left[t_{c-1}\right]$ and $\chi_{D_{k}}^{M}\left[t_{c-1}\right]$ are $\varepsilon_{D_{k}}^{M}$ and $\chi_{D_{k}}^{M}$ values for $\mathscr{D}_{k}$ at the time $t_{c-1} \cdot \varepsilon_{D_{e}}^{M_{i}}\left[t_{c-2}\right]$ and $\chi_{D_{e}}^{S_{j}}\left[t_{c-2}\right]$ are $\varepsilon_{D_{e}}$ and $\chi_{D_{e}}^{M_{i}}$ values for $\mathscr{D}_{e}$ at the time $t_{c-2}$. By considering the traffic flow balance between $\mathscr{D}_{e}$ and $\mathscr{D}_{k}$, the function $F\left(\mathscr{D}_{e}, \mathscr{D}_{k}\right)$ is designed based on the repeated bargaining concept. Therefore, the outcome of $F\left(\mathscr{D}_{e}, \mathscr{D}_{k}\right)$ is dynamically adjusted based on the timed online manner for networkassisted D2D communications.

\subsection{Main Steps of Proposed DC-Based Spectrum Allocation} Scheme. In this study, we develop a novel spectrum allocation scheme for DC-based D2D communications. It is designed based on the joint-bargaining game model to reach a fine solution. Based on the hierarchical interconnection of spectrum allocation processes, control decisions can cause cascade interactions in a distributed interactive manner. In the proposed scheme, the NBS, WNBS, and NBS-CS are mutually dependent to strike the appropriate performance balance for the HetNet system. Therefore, intelligent network agents interact with each other and work together through the dynamics of our hierarchical feedback mechanism. Usually, conventional optimization methods such as Lagrangian or dynamic programming require global objective functions with exponential time complexity; it is impractical to be implemented for realistic system operations. However, our joint-bargaining process is designed based on the hierarchical HetNet platform to reduce computational complexity. Therefore, decision mechanism is implemented with polynomial complexity; it is an important feature of the proposed scheme. The main steps of the proposed scheme can be described as follows (Figure 1):

Step 1. For our simulation model, the values of system parameters and control factors can be discovered in Table 1, and the simulation scenario is given in Section 4.

Step 2. In each time period, individual MDs generate their application services and contact their corresponding BSs. Individual SBSs report their traffic request amounts $(r)$ to their corresponding MBSs.

Step 3. In the first phase, each individual MBS $(M)$ shares its spectrum resource $\left(\mathscr{M}_{M}\right)$ with the set of SBSs $\left(\mathscr{W}_{M}\right)$. Based on the concept of NBS, $\mathscr{M}_{M}$ is distributed to multiple $S \in \mathscr{W}_{M}$, and each $S$ can get its spectrum resource $\left(\chi_{S}^{M}\right)$ according to $(4)$.

Step 4 . In the second phase, each individual $S$ allocates its assigned spectrum pool $\left(\chi_{S}^{M}\right)$ for its corresponding MDs in $\mathbf{N}_{S}^{M}$. While prioritizing network-assisted D2D communications, $\chi_{S}^{M}$ is distributed for the 


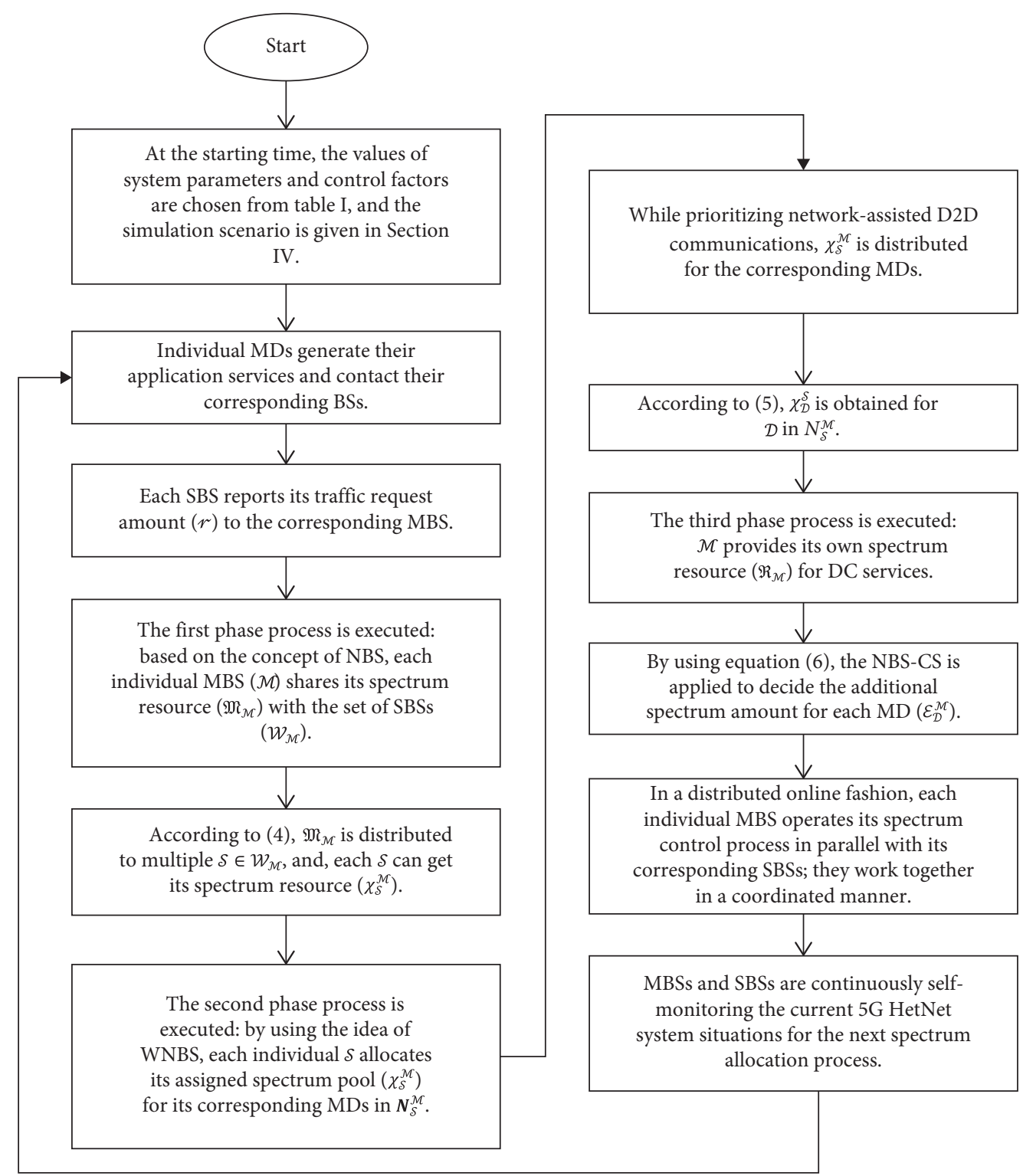

FIgURE 1: Flowchart 1: flowchart table of the proposed spectrum allocation algorithm.

corresponding MDs by using the idea of WNBS. For $\mathscr{D}$ in $\mathbf{N}_{S}^{M}, \chi_{D}^{S}$ is obtained according to (5).

Step 5. In the third phase, $M$ provides its own spectrum resource $\left(\mathfrak{R}_{M}\right)$ for DC services. $\mathbf{N}_{C}^{M_{i}}$ and $\mathbf{N}_{D}^{M_{i}}$ are treated as a unit, and the NBS-CS is applied to decide the additional spectrum amount for each $\mathrm{MD}\left(\varepsilon_{D}^{M}\right)$; it is obtained by using equation (6).

Step 6. In a distributed online fashion, each individual MBS operates its spectrum control process in parallel with its corresponding SBSs, which are performing individually their spectrum control processes.

Step 7. In the hierarchical HetNet platform, MBSs and SBSs work together in a coordinated manner to strike the appropriate performance balance between contradictory requirements.
Step 8. Constantly, the MBSs and SBSs are self-monitoring the current 5G HetNet system situations and proceed to Step 2 for the next spectrum allocation process.

\section{Performance Evaluation}

In this section, the performance of our proposed scheme is evaluated by simulations, and it is compared with other existing protocols to confirm the superiority of our jointbargaining approach. As mentioned in Section 2, we select the NRBL, SQSI, and SDRA schemes $[1,13,14]$; these existing schemes are recently published D2D communication protocols for the HetNet system platform. The assumptions of our simulation environments are as follows: 
TABLE 1: System parameters used in the simulation experiments.

\begin{tabular}{|c|c|c|}
\hline Parameter & Value & Description \\
\hline$n$ & 16 & The number of MBSs in the HetNet system \\
\hline$m$ & 64 & The number of SBSs in the HetNet system \\
\hline$k$ & 100 & The number of MDs \\
\hline $\mathfrak{R}_{M}$ & 2 giga bps & M's own spectrum resource \\
\hline $\mathscr{M}_{M}$ & 8 giga bps & The spectrum resource for SBSs in $M$ \\
\hline$r_{M}$ & $3 \mathrm{~km}$ & The radius of MBS's coverage area \\
\hline$r_{S}$ & $1.5 \mathrm{~km}$ & The radius of SBS's coverage area \\
\hline$\Psi_{S}$ & 0.95 & A profit coefficient factor for $U_{S}^{M}(\cdot)$ \\
\hline$\mu$ & 0.3 & A control parameter for $U_{S}^{M}(\cdot)$ \\
\hline$\varrho$ & 1 & Bargaining power for simple cellular MDs \\
\hline$\beta$ & 1.2 & Bargaining power for network-assisted D2D MDs \\
\hline$\kappa, \eta, \xi$ & $2,1.5,1$ & Control parameters to evaluate $U_{D}$ for single $\mathrm{MD}$ \\
\hline$\Gamma$ & 1 & A control parameter for $U_{D}^{S}$ \\
\hline$\alpha$ & 1 & A profit coefficient factor for $F(D)$ when $D \notin \mathscr{X}_{D 2 D}$ \\
\hline$\psi$ & 1.25 & A profit coefficient factor for $F(D)$ when $D \in \in \mathscr{X}_{D 2 D}$ \\
\hline$\phi$ & 0.3 & A control parameter for $F(D)$ when $D \in \in \mathscr{X}_{D 2 D}$ \\
\hline Applications & Bandwidth requirement & Connection duration \\
\hline Application 1 & $10 \mathrm{Mbps}$ & $180 t$-unit \\
\hline Application 2 & $7.5 \mathrm{Mbps}$ & $90 t$-unit \\
\hline Application 3 & $5 \mathrm{Mbps}$ & $30 t$-unit \\
\hline Application 4 & $2.5 \mathrm{Mbps}$ & $60 t$-unit \\
\hline
\end{tabular}

(i) The simulated HetNet system platform consists of two tiers, where $16 \mathrm{MBSs}$ are in the upper tier and 64 SBSs are in the lower tier.

(ii) Multiple BSs are regularly positioned in an area of $10 \times 10$-kilometer square area; MBS's radius $r_{M}$ and SBS' radius $r_{M}$ of their coverage areas are 3 and 1.5 kilometers, respectively.

(iii) There are one hundred MDs $\mathscr{D}=\left\{D_{1}, \ldots, D_{100}\right\}$, and they are distributed randomly over the HetNet cellular area.

(iv) The process for service request generations is Poisson with rate $\Lambda$ (services/s), and the range of offered service load was varied from 0 to 3.0.

(v) Four different kinds of applications are assumed based on connection duration and spectrum requirement. In each $\mathrm{MD}$, applications are generated randomly.

(vi) Among the generated different kinds of applications, some applications are paired for networkassisted D2D communications. They are also randomly selected.

(vii) The spectrum capacities of $\mathfrak{R}_{M}$ and $\mathscr{M}^{M}$ are 2 Giga bps and 8 Giga bps, respectively.

(viii) To reduce computation complexity, the amount of spectrum allocation is specified in terms of basic spectrum units (BSUs), where one BSU is the minimum amount (e.g., $512 \mathrm{kbps}$ in our system) of spectrum adjustment.

(ix) To calculate the bargaining solutions, the utilities of disagreement points, that is, $d_{S}$ and $d_{D}$ are zeros in our system. (x) System performance measures obtained on the basis of 100 simulation runs are plotted as a function of the offered service request load.

(xi) Performance measures obtained are normalized user's payoff, system throughput, and fairness among BSs in the HetNet system.

(xii) For simplicity, we assume the absence of physical obstacles in the wireless communications.

In Figure 2, the normalized MD's payoff is investigated for various service request rates. In this figure, it can be seen that the curves represent the utility payoff assigned for MDs in the HetNet system. In the users' point of view, this performance criterion is related to end user's satisfaction and service quality. In our proposed scheme, the idea of joint-bargaining solutions is applied, and each BS may hierarchically provide the available spectrum resource for its corresponding MDs while maximizing MDs' payoffs. It can lead to higher MD's payoff by adapting the current HetNet system. As expected, we observe that our joint-bargaining approach has a comparatively better MD's payoff than the other existing NRBL, SQSI, and SDRA schemes under lightto-heavy service request load distributions.

In order to examine the performance of our networkassisted D2D technique with respect to the different service request rates, we plot the system throughput in Figure 3. In our simulation model, the system throughput is estimated as the ratio of traffic service that is successfully completed to all requested applications. In our proposed scheme, the limited spectrum resource is fair-efficiently shared to induce selfish MDs to operate their services. As a consequence of iterative three-step bargaining process, BSs can adapt the current network conditions and effectively allocate the spectrum 


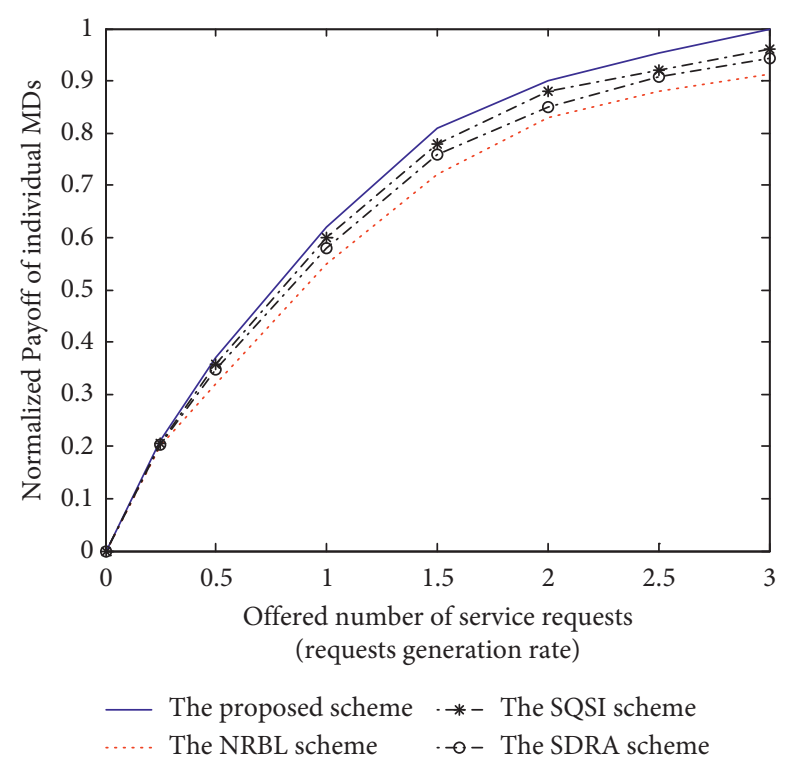

Figure 2: Normalized payoff of individual MDs.

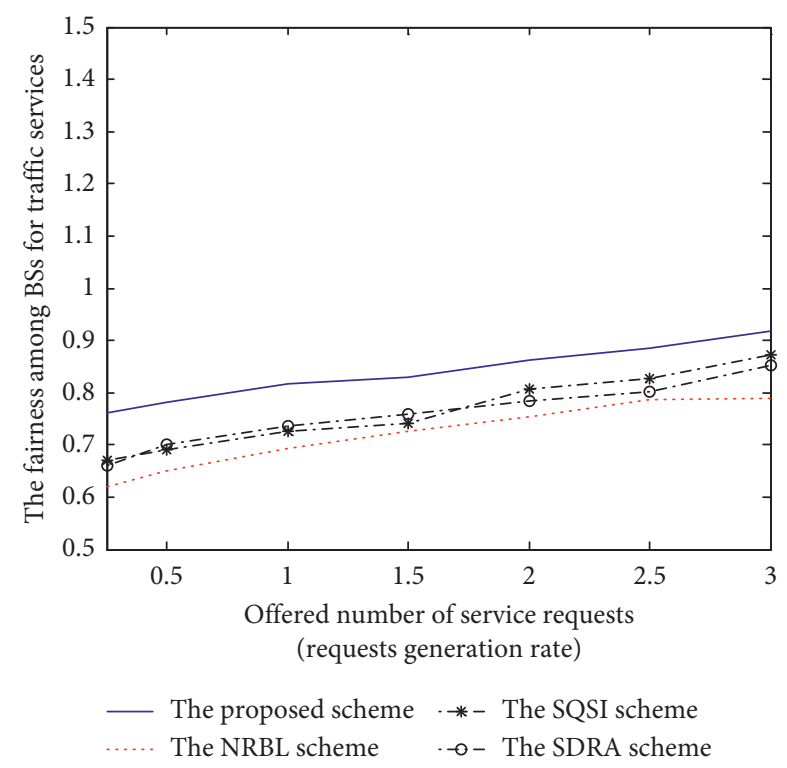

FIgure 3: The fairness among BSs for traffic services.

resource to maximize the system throughput. Under different service request intensities, the performance trend shown in Figure 3 is very similar to the curves in Figure 4. Thus, similar conclusions to the ones of Figure 4 are reached.

In order to study the impact of our joint-bargaining game model, we plot the achieved fairness among BSs for requested services of MDs. To compare the fairness performance, we use Jain's fairness index [17]; it varies between one and zero and represents how to effectively share the limited spectrum resources among BSs. Lower Jain's fairness index indicates lower fairness in the BSs' spectrum allocations. Our three-step bargaining game model effectively compromises the contrasting viewpoints of different MDs and provides the most proper spectrum sharing protocol.

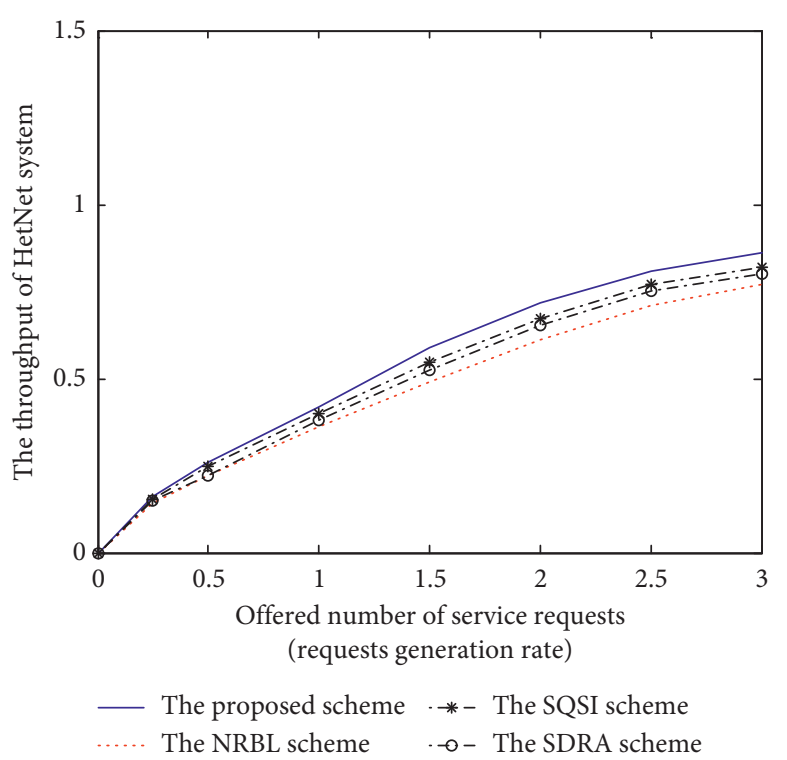

FIgURE 4: Throughput of HetNet system.

Therefore, under diversified traffic condition changes, our proposed scheme can maintain a higher fairness index than the existing NRBL, SQSI, and SDRA schemes. From the simulation result in Figures 1-3, it is evident that, in general, our joint-bargaining game mechanism has a big impact on the performance for the HetNet system in terms of MD's payoff, system throughput, and fairness.

\section{Summary and Conclusions}

Maintaining dual wireless connections is a promising solution to boost capacity in 5G HetNets, where MDs are able to consume spectrum resources of dual serving cells simultaneously. In this paper, the issues of network-assisted D2D communications have been studied based on the emerging dual-connectivity paradigm. This combined approach can be regarded as an attractive access method while significantly enhancing the network performance and QoS. However, designing such integrated mechanism is challenging due to the following problems; (i) the spectrum distribution problem for different SBSs, (ii) the spectrum allocation problem for multiple MDs, and (iii) the spectrum sharing problem for $\mathrm{D} 2 \mathrm{D}$ pairs. To effectively handle these control problems, we adopt the basic Nash ideas and employ three bargaining solutions, NBS, WNBS, and NBS-CS, to design our spectrum control scheme. By taking into account the hierarchical HetNet system platform, different bargaining solutions are sophisticatedly combined into the holistic scheme and act cooperatively and collaborate with each other in a distributed manner. Therefore, the limited spectrum resources are effectively shared among different BSs and multiple MDs under dynamically changing HetNet environments. Through numerical simulation analysis, we have illustrated that the proposed scheme achieves better performance as compared to the existing NRBL, SQSI, and SDRA protocols. Therefore, we can confirm the effectiveness of our joint-bargaining approach when the network-assisted 
D2D communication and DC technique coexist in the HetNet system.

For the future work, our current study can be extended in a number of ways. One future direction is to design a framework based on the cloud radio access network technology and the control-data decoupled architecture. Another potential direction for the future research is to aim at maximizing the energy efficiency by optimizing jointly the traffic load prediction, the cell association, and the dynamic power strategies with respect to the time-varying traffic load. In addition, we will construct a stochastic optimization problem of handover overhead with queue backlog, under the condition of ensuring user fairness and limited resource constraints.

\section{Data Availability}

The data are available upon request to the corresponding author at swkim01@sogang.ac.kr.

\section{Conflicts of Interest}

The author declares that there are no conflicts of interest regarding the publication of this paper.

\section{Authors' Contributions}

The author participated in the design of the study and performed the statistical analysis.

\section{Acknowledgments}

This research was supported by the MSIT (Ministry of Science and ICT), Republic of Korea, under the ITRC (Information Technology Research Center) support program (IITP-2020-2018-0-01799) supervised by the IITP (Institute for Information \& Communications Technology Planning \& Evaluation), and was supported by Basic Science Research Program through the National Research Foundation of Korea (NRF) funded by the Ministry of Education (NRF-2018R1D1A1A09081759).

\section{References}

[1] S. W. H. Shah, A. N. Mian, and J. Crowcroft, "Statistical qos guarantees for licensed-unlicensed spectrum interoperable D2D communication," IEEE Access, vol. 8, pp. 27277-27290, 2020.

[2] G. S. Park and H. Song, "Video quality-aware traffic offloading system for video streaming services over $5 \mathrm{G}$ networks with dual connectivity," IEEE Transactions on Vehicular Technology, vol. 68, no. 6, pp. 5928-5943, 2019.

[3] X. Jia, Q. Fan, W. Xu, and L. Yang, "Cross-tier dual-connectivity designs of three-tier hetnets with decoupled uplink/downlink and global coverage performance evaluation," IEEE Access, vol. 7, pp. 16816-16836, 2019.

[4] M. Hasan and E. Hossain, "Distributed resource allocation for relay-aided device-to-device communication: a message passing approach," IEEE Transactions on Wireless Communications, vol. 13, no. 11, pp. 6326-6341, 2014.
[5] G. Fodor, E. Dahlman, G. Mildh et al., "Design aspects of network assisted device-to-device communications," IEEE Communications Magazine, vol. 50, no. 3, pp. 170-177, 2012.

[6] A. Maria, E. Pardo, O. Galinina, S. Andreev, and M. Dohler, "Flexible dual-connectivity spectrum aggregation for decoupled uplink and downlink access in 5G heterogeneous systems," IEEE Journal on Selected Areas in Communications, vol. 34, no. 11, pp. 2851-2865, 2016.

[7] S. Kim, Game Theory Applications in Network Design, IGI Global, Hershey, PA, U.S.A, 2014.

[8] G. Bergantiños, B. Casas-Méndes, M. G. Fiestras-Janeiroand Juan, and J. Vidal-Puga, "A focal-point solution for bargaining problems with coalition structure," University Library of Munich, Munich, Germany, 2005.

[9] S. Chae and P. Heidhues, "A group bargaining solution," Mathematical Social Sciences, vol. 48, no. 1, pp. 37-53, 2004.

[10] X. Wang, S. Kwong, L. Xu, and Y. Zhang, "Generalized nash bargaining solution to rate control optimization for spatial scalable video coding," IEEE Transactions on Image Processing, vol. 23, no. 9, pp. 4010-4021, 2014.

[11] X. Liu and X. Zhang, "NOMA-based resource allocation for cluster-based cognitive industrial internet of things," IEEE Transactions on Industrial Informatics, vol. 16, no. 8, pp. 5379-5388, 2020.

[12] X. Liu, Su Hu, M. Li, and B. Lai, "Energy-efficient resource allocation for cognitive industrial Internet of Things with wireless energy harvesting," IEEE Transactions on Industrial Informatics, 2020.

[13] S. Maghsudi and S. Stanczak, "Channel selection for networkassisted D2D communication via no-regret bandit learning with calibrated forecasting," IEEE Transactions on Wireless Communications, vol. 14, no. 3, pp. 1309-1322, 2015.

[14] Y. Liu, Y. Wang, R. Sun, and Z. Miao, "Distributed resource allocation for $\mathrm{d} 2 \mathrm{~d}$-assisted small cell networks with heterogeneous spectrum," IEEE Access, vol. 7, pp. 83900-83914, 2019.

[15] K. Long, P. Wang, W. Li, and D. Chen, "Spectrum resource and power allocation with adaptive proportional fair user pairing for noma systems," IEEE Access, vol. 7, pp. 8004380057, 2019.

[16] L. $\mathrm{Pu}, \mathrm{X}$. Chen, J. Xu, and $\mathrm{X}$. Fu, "D2D fogging: an energyefficient and incentive-aware task offloading framework via network-assisted D2D collaboration," IEEE Journal on Selected Areas in Communications, vol. 34, no. 12, pp. 38873901, 2016.

[17] M. Dianati, X. Shen, and S. Naik, "A new fairness index for radio resource allocation in wireless networks," in Proceedings of the IEEE Wireless Communications and Networking Conference, pp. 712-715, New Orleans, LA, USA, March 2005. 